\title{
The Anomalous X-ray Pulsar 4U 0142+61: Variability in the infrared and a spectral break in the optical ${ }^{\star}$
}

\author{
F. Hulleman ${ }^{1}$, M. H. van Kerkwijk ${ }^{1, \star \star}$, and S. R. Kulkarni ${ }^{2}$ \\ 1 Astronomical Institute, Utrecht University, PO Box 80000, 3508 TA Utrecht, The Netherlands \\ 2 Palomar Observatory, California Institute of Technology 105-24, Pasadena, CA 91125, USA
}

Received 10 January 2003 / Accepted 2 December 2003

\begin{abstract}
We present new optical and infrared observations of the counterpart to the Anomalous X-ray Pulsar (AXP) 4U 0142+61 taken with the Keck I telescope. The counterpart is found to be variable in the infrared. This contrasts with our optical observations, which do not show any evidence for variability. Apart from the variability the AXP shows a remarkable spectral energy distribution. In particular, we find a sudden drop in flux going from $V$ to $B$, presumably due to a spectral feature. We compare our results to those obtained for the two other securely identified AXP counterparts, to 1E 2259+586 and 1E 1048.1-5937. 4U 0142+61 is very similar to the former source in its X-ray timing and spectral properties, and we find that this similarity extends to the quiescent infrared to X-ray flux ratio. For 1E 1048.1-5937, which has different X-ray properties, the situation is less clear: in one observation, the infrared to X-ray flux ratio was much larger, but another observation gave an upper limit which is consistent with that observed for $4 \mathrm{U} 0142+61$. Assuming the quiescent ratios are all similar, we estimate the optical and infrared brightnesses for the three AXPs that remain to be identified as well as for the four Soft Gamma-ray Repeaters. We also discuss briefly how the observed optical and infrared emission might arise, in particular in the context of the magnetar model.
\end{abstract}

Key words. pulsars: individual: 4U 0142+61 - stars: neutron - stars: magnetic fields - X-rays: general

\section{Introduction}

Recent developments have shown that the neutron star population is much more diverse than previously thought. For example, increasing numbers of $\mathrm{X}$-ray point sources are found in or near the centers of supernova remnants (SNR) that do not display any of the usual signs of (radio) pulsar activity (Pavlov et al. 2002).

Particularly interesting in this regard are the so-called Anomalous X-ray Pulsars (AXPs; see Israel et al. 2002b, for a recent review); and Soft Gamma Repeaters (SGRs), which are believed to be magnetars (Duncan \& Thompson 1992; Thompson \& Duncan 1996), neutron stars whose emission is powered by decay of ultra-strong magnetic fields.

For the AXPs, this belief rests mainly on the failure to explain them using the conventional power sources, rotational energy loss and accretion. The former fails because the

Send offprint requests to: F. Hulleman,

e-mail: F.Hulleman@astro.uu.nl

* Table 3 is only available in electronic form at the CDS via anonymous ftp to

cdsarc.u-strasbg.fr $(130.79 .128 .5)$ or via

http://cdsweb.u-strasbg.fr/cgi-bin/qcat?J/A+A/416/1037

$\star \star$ Current Address: Dept. of Astronomy \& Astrophysics, 60

St George St., Toronto, ON, M5S 3H8, Canada.

e-mail: mhvk@astro.utoronto.ca spin-down energy implied by the period and its derivative $\left(L_{\mathrm{sd}} \simeq 10^{32} \mathrm{ergs} \mathrm{s}^{-1}\right)$ is much less than the energy emitted in $\mathrm{X}$-rays alone. The strongest evidence against accretion is the faintness of the optical counterparts, which sets strong limits on the size of an accretion disk (e.g., Hulleman et al. 2000a, hereafter Paper I). Moreover, the high pulsed fraction (27\%) of the optical pulsations of AXP 4U 0142+61 (Kern \& Martin 2002), seems inconsistent with reprocessing of X-rays.

Two of the five sources are associated with supernova remnants (Gaensler et al. 2001), indicating that AXPs are young objects. For none of the sources, there is any evidence for orbital motion (from timing, e.g., Wilson et al. 1999; Mereghetti et al. 1998).

Recently, great progress has been made observationally: better timing (RXTE, e.g., Gavriil \& Kaspi 2002), improved positional accuracy (Chandra, e.g., Hulleman et al. 2001; Wang \& Chakrabarty 2002), higher spectral resolution (Chandra and $X M M$, e.g., Patel et al. 2001; Juett et al. 2002; Tiengo et al. 2002), and the discovery of optical/IR counterparts (Paper I; Hulleman et al. 2001; Wang \& Chakrabarty 2002; Israel et al. 2003) Moreover, SGR-like X-ray bursts from two AXPs (1E 1048.1-5937, Gavriil et al. 2002; 1E 2259+586, Kaspi et al. 2003) have been detected, providing strong evidence for a common nature of AXPs and SGRs.

These observations have spurred theoretical work. Much effort has gone into the development of model atmospheres 


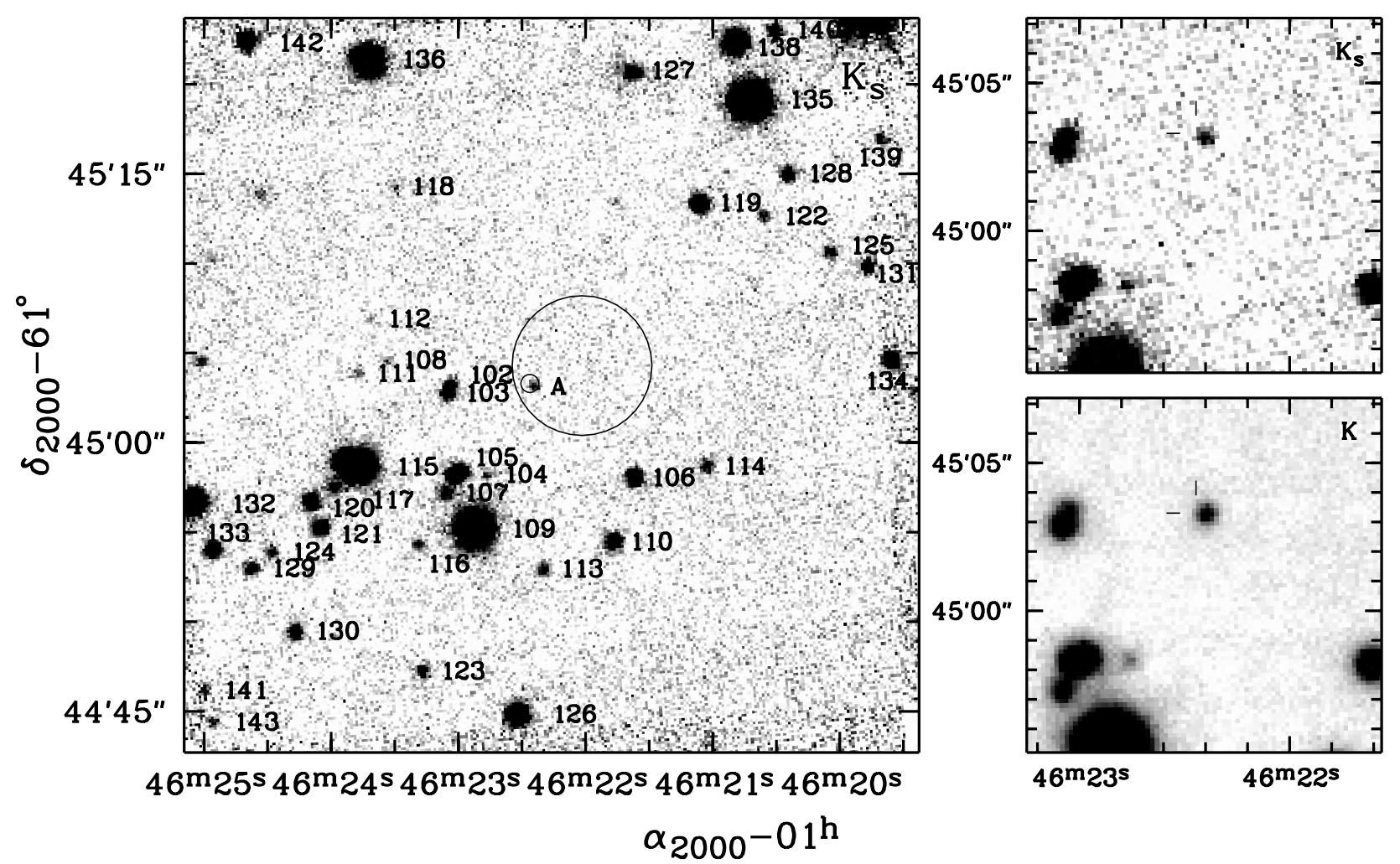

Fig. 1. Left: $K_{\mathrm{s}}$ band mosaic of the field around AXP 4 U 0142+61. Overdrawn are the Einstein (White et al. 1987) and Chandra (Juett et al. 2002) error circles and the identifications of the stars listed in Table 3. Right: close ups of the 93 min K and 9 min $K_{\mathrm{s}}$ band mosaics with the Chandra position indicated by tick marks.

for strongly magnetized neutron stars (e.g. Heyl \& Hernquist 1998; Zane et al. 2001; Özel 2001; Ho \& Lai 2003), which help to understand the blackbody-like (thermal) component of the X-ray spectrum, as well as in models for magnetar magnetospheres, which may help to understand the power-law (non-thermal) component (Thompson et al. 2002).

Observations at optical and infrared (IR) wavelengths provide important clues about the origin of the non-thermal emission and thus of the physical conditions in magnetar magnetospheres. In this paper we focus on the optical/IR spectral energy distribution of $4 \mathrm{U} 0142+61$, the X-ray brightest of the AXPs.

In Sect. 2, we start with a description of the new optical and infrared observations we obtained. Then, in Sect 3, we discuss the reddening to the source and derive the spectral energy distribution. We make a comparison with two other secure counterparts, to AXPs 1E 2259+586 and 1E 1048.1-5937, and give predictions for the optical/infrared fluxes of other magnetars in Sect. 4. We end with a discussion in Sect. 5.

\section{Observations}

\subsection{Near infrared photometry}

Infrared images in the $K$ band were obtained on the nights of 1999 February 7 and 8 with the Near Infrared Camera (NIRC, Matthews \& Soifer 1994), mounted on the Keck I telescope. During both nights the conditions were photometric, and the seeing was good at $0 . ' 6$ to $0 .{ }^{\prime} 8$.
A total of 54 frames were taken on the first night and 39 on the second night. Each frame consisted of 4 co-added exposures of $15 \mathrm{~s}$ integration each and hence the total exposure times were 54 and $39 \mathrm{~min}$. After subtraction of a dark frame, we created a flatfield, using the observations themselves, and a mosaic, for each night separately, in the same way as described in Hulleman et al. (2001). In Fig. 1 we show a close-up of the average of the mosaics of the two nights. The individual mosaics are of comparable quality.

Further images were obtained on 2001 October 31 in the $K_{\mathrm{s}}$ band. The conditions were again photometric, and the seeing was excellent at 0 . $^{\prime} 4$. We took frames consisting of five coadded exposures of $10 \mathrm{~s}$ each. A mosaic was created consisting of 11 such frames, giving a total exposure time of $550 \mathrm{~s}$. The results is also shown in Fig. 1.

Finally, an additional set of 18 frames, each consisting of five co-added exposures of $10 \mathrm{~s}$, was obtained on 2002 December 18. Again, conditions were photometric and the seeing excellent at 0.5 . The resulting mosaic is similar to that in Fig. 1 and is not shown.

Photometry was performed using DAOPHOT II as implemented in the ESO-MIDAS software package. Absolute calibration was achieved in the following way. First a co-add was taken of a standard star from the list of Persson et al. (1998), followed by a co-add with a position offset such that it only contained sky. The latter image was then subtracted from the former to produce a corrected image on which photometry was done using a large aperture. An aperture correction was 
Table 1. Infrared observations taken with NIRC on Keck I.

\begin{tabular}{|c|c|c|c|c|}
\hline Field & Band & Date & $\begin{array}{l}\text { exposure } \\
\text { (s) }\end{array}$ & Seeing \\
\hline $4 \mathrm{U} 0142+61$ & $\bar{K}$ & 1999 Feb. 07 & $54 \times 4 \times 15$ & 0.7 \\
\hline SJ 9134 & $K$ & 1999 Feb. 07 & $10 \times 0.4$ & 0.7 \\
\hline $4 \mathrm{U} 0142+61$ & $K$ & 1999 Feb. 08 & $39 \times 4 \times 15$ & 0.7 \\
\hline SJ 9108 & $K$ & 1999 Feb. 08 & $10 \times 0.4$ & 0.7 \\
\hline $4 \mathrm{U} 0142+61$ & $K_{\mathrm{s}}$ & 2001 Oct. 30 & $11 \times 5 \times 10$ & 0.4 \\
\hline SJ 9126 & $K_{\mathrm{s}}$ & 2001 Oct. 30 & $4 \times 10 \times 0.4$ & 0.9 \\
\hline SJ 9134 & $K_{\mathrm{s}}$ & 2001 Oct. 30 & $2 \times 10 \times 0.4$ & $0 . .4$ \\
\hline $4 \mathrm{U} 0142+61$ & $K_{\mathrm{s}}$ & 2002 Dec. 18 & $18 \times 5 \times 10$ & 0.5 \\
\hline
\end{tabular}

determined using several bright stars on the science frame. Finally, we corrected for atmospheric extinction, using the median value of $0.088 \mathrm{mag}^{\text {airmass }}{ }^{-1}$ at the Mauna Kea summit (from an extension ${ }^{1}$ of the work by Krisciunas et al. 1987).

On both 1999 February 7 and 8 only a single co-add of a standard star was taken, using SJ 9134 and SJ 9108 respectively. Unfortunately the results for the two nights disagree by $0.17 \mathrm{mag}$ in zero-point. For $4 \mathrm{U} 0142+61$, using the mean of the magnitudes determined for both nights, we find $K=19.64 \pm 0.04 \pm 0.17 \mathrm{mag}$, where the latter error reflects the uncertainty in the zero-point (but see below).

On 2001 October 31 multiple standard star co-adds were taken, viz. four co-adds of standard SJ 9126 and two co-adds of SJ 9134. For both standards we used the mean of the images to fix the zero point. The agreement between the two standards was very good, the difference being only $0.002 \mathrm{mag}$. Using the zero point, airmass and aperture corrections to correct the instrumental (DAOPHOT) magnitude, we find $K_{\mathrm{s}}=20.15 \pm 0.08$ for the AXP. This is 0.5 mag fainter than in $K$ !

We verified that this was not due to a zero-point error by measuring the difference between the $K$ and $K_{\mathrm{s}}$ magnitudes derived for all objects, other than the AXP. This difference is found to be $-0.039 \pm 0.020 \mathrm{mag}$, i.e. within the estimated zero-point uncertainty of $0.17 \mathrm{mag}$ in the 1999 data. Since the $K_{\mathrm{s}}$-band zero-point is much better determined, we have used it to correct the $K$ magnitudes. The revised $K$ magnitude for $4 \mathrm{U} 0142+61$ is $K=19.68 \pm 0.05 \mathrm{mag}$ (including the uncertainty in the corrected zero point).

For the December 2002 data we again used the calibration obtained from the October 2001 data. For all objects, except the AXP, for which $K_{\mathrm{s}}=19.85 \pm 0.04$, we find consistent results. Clearly, the counterpart of $4 \mathrm{U} 0142+61$ is variable. We return to this in Sect. 3.

Finally, we note that when we compared our results with the photometry of Coe \& Pightling (1998), we found that our magnitudes are $\sim 0.5 \mathrm{mag}$ brighter than theirs. We do not understand the reason for this discrepancy. We considered whether it might be due to the fact that the objects in common are rather bright in our images, and hence might be affected by detector non-linearities. If so, one would expect the $K$ and $K_{\mathrm{s}}$ band results to be different for the brighter stars, since the $K_{\mathrm{s}}$ band is less wide than the $K$ band and the individual exposures in $K_{\mathrm{s}}$

\footnotetext{
${ }^{1}$ http://www2.keck.hawaii.edu/inst/nirc/exts.html
}

were shorter than in $K$. Comparing our $K$ and $K_{\mathrm{s}}$ magnitudes, we indeed find that the brightest objects have somewhat larger differences than the typical ones, but these differences are still less than about $0.05 \mathrm{mag}$ and hence cannot explain the discrepancy with the results of Coe \& Pightling (1998).

\subsection{Optical photometry}

Optical observations were obtained with the Low Resolution Imaging Spectrograph (LRIS, Oke et al. 1995) at either of the two Keck telescopes. For photometric calibration, we also used observations taken with the 60-inch telescope on Palomar mountain, using the direct imaging camera at the Cassegrain focus. A summary of the observations is given in Table 2.

Observations in the $B$ band were made for us on 2000 September 3 . The conditions were photometric and the seeing was good at 0.7 . Six dithered exposures of $600 \mathrm{~s}$ each were taken. During this night, the usual LRIS CCD was not available, and a spare was used.

On 2000 December 29, further LRIS observations were taken, in $B$ and $R$. The conditions were photometric and the seeing was $0 . ' 8$ in both $B$ and $R$. Unfortunately, during all but one of the $R$-band exposures of the AXP the guider failed. Consequently the $R$ band image is of poorer quality than the one presented in Paper I. Standard data reduction procedures were used (see description in Hulleman et al. 2000a).

Parts of the averaged $B$-band images are shown in Fig. 2.

\subsubsection{Photometric calibration}

For calibration purposes, additional images of the field of $4 \mathrm{U} 0142+61$ and of standard fields were taken with the 60 inch telescope on Palomar mountain. For details, see Table 2. On 2000 July $23, V, R$ and $I$-band images were taken under photometric conditions, with seeing of about 1 '. 3 . These images were used for the calibration in Paper I. In order to verify the calibration, as well as to obtain a calibration for the $B$ band, additional images were taken on 2000 November $18(B, V, R$ and $I)$ and $19(B$ and $V)$. During both nights, the conditions were photometric, but the seeing was very bad (4!'0) on the 18 th, and poor $(2$.' 1$)$ on the 19 th.

Positions and magnitudes of standard stars were taken from tables obtained from the $\mathrm{WWW}^{2}$ (Stetson 2000), that were last updated on 2001 June 28. Instrumental magnitudes for these stars were obtained using aperture photometry with the DAOPHOT II package (Stetson 1987). For the 2000 Nov. 18 images, which had the worst seeing, we found that so few standard stars were detected that we could use this set only for consistency checks.

The 2000 July 23 images of NGC 7790, PG 1657+078 and $4 \mathrm{U} 0142+61$ were all taken at comparable airmass, and hence no extinction correction was needed. Small but significant colour terms were found for all bands $(V, R$ and $I)$. With these corrections, the estimated uncertainties in the zero point are about $0.02 \mathrm{mag}$.

\footnotetext{
${ }^{2}$ http://cadcwww.hia.nrc.ca/standards/
} 
Table 2. Optical observations taken at Keck and the Palomar 60-inch.

\begin{tabular}{|c|c|c|c|c|c|c|}
\hline field & instrument & bands & date & total exposure (s) & seeing & reference \\
\hline $4 \mathrm{U} 0142+61$ & KeckI/LRIS & $V R I$ & 1994 Oct. 31 & $1500,1800,200$ & $1{ }^{\prime \prime} 0$ & Paper I \\
\hline $4 \mathrm{U} 0142+61$ & KeckII/LRIS & $R I$ & 1999 Sep. 06 & 3000,1380 & 0.6 & Paper I \\
\hline PG $0231+051$ & KeckII/LRIS & $R I$ & 1999 Sep. 06 & 4,4 & $a$ & Paper I \\
\hline PG $1657+078$ & $\mathrm{P} 60 / \mathrm{CCD} 13$ & $R I V$ & 2000 Jul. 23 & $130,120,165$ & 1.9 & This work \\
\hline NGC 7790 & $\mathrm{P} 60 / \mathrm{CCD} 13$ & $R I V$ & 2000 Jul. 23 & $360,360,360$ & $1 . " 3$ & This work \\
\hline $4 \mathrm{U} 0142+61$ & $\mathrm{P} 60 / \mathrm{CCD} 13$ & $R V I$ & 2000 Jul. 23 & $1230,1050,330$ & $1 . " 3$ & This work \\
\hline NGC 7790 & $\mathrm{P} 60 / \mathrm{CCD} 13$ & $V R I$ & 2000 Jul. 23 & $30,20,30$ & $1 . " 3$ & This work \\
\hline 4U 0142+61 & KeckI/LRIS & $B$ & 2000 Sep. 03 & 3600 & 0.7 & This work \\
\hline NGC 7654 & $\mathrm{P} 60 / \mathrm{CCD} 13$ & $B V R I$ & 2000 Nov. 18 & $30,30,30,30$ & 2.3 & This work \\
\hline NGC 7790 & $\mathrm{P} 60 / \mathrm{CCD} 13$ & $B V R I$ & 2000 Nov. 18 & $30,30,30,30$ & 1.9 & This work \\
\hline Landolt 92 & $\mathrm{P} 60 / \mathrm{CCD} 13$ & $B V R I$ & 2000 Nov. 18 & $30,30,30,30$ & $5{ }^{\prime \prime} 0$ & This work \\
\hline 4U $0142+61$ & $\mathrm{P} 60 / \mathrm{CCD} 13$ & $B V R I$ & 2000 Nov. 18 & $330,330,330,330$ & $4 . .0$ & This work \\
\hline NGC 2419 & $\mathrm{P} 60 / \mathrm{CCD} 13$ & $B V R I$ & 2000 Nov. 18 & $120,120,120,120$ & $3 . \prime 5$ & This work \\
\hline NGC 7654 & $\mathrm{P} 60 / \mathrm{CCD} 13$ & $B V R I$ & 2000 Nov. 19 & $30,30,30,30$ & $2 . .5$ & This work \\
\hline NGC 7790 & $\mathrm{P} 60 / \mathrm{CCD} 13$ & $I R B V$ & 2000 Nov. 19 & $30,30,30,30$ & $2 . .7$ & This work \\
\hline PG $0231+051$ & $\mathrm{P} 60 / \mathrm{CCD} 13$ & $B V R I$ & 2000 Nov. 19 & $30,30,30,30$ & 2.3 & This work \\
\hline 4U 0142+61 & $\mathrm{P} 60 / \mathrm{CCD} 13$ & $B V$ & 2000 Nov. 19 & 330,330 & 2.1 & This work \\
\hline PG $0231+051$ & $\mathrm{P} 60 / \mathrm{CCD} 13$ & $R I V B$ & 2000 Nov. 19 & $30,30,30,30$ & $2 . .7$ & This work \\
\hline $4 \mathrm{U} 0142+61$ & KeckI/LRIS & $B R$ & 2000 Dec. 29 & 3500,1050 & $0 . ! 8$ & This work \\
\hline
\end{tabular}

${ }^{a}$ These images were deliberately taken out of focus.

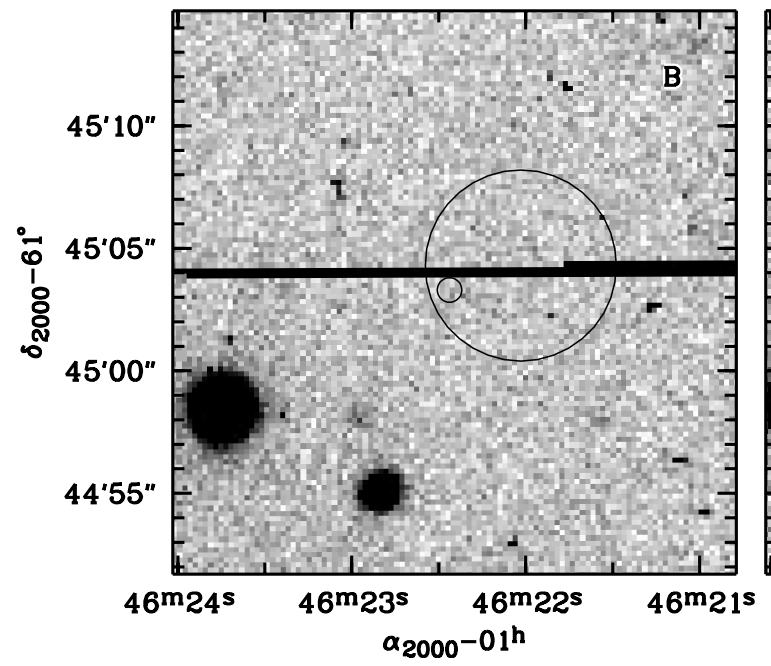

For the 2000 Nov. 19 data, there was a problem, in that the zero points inferred from the the NGC 7654 field differed by 0.2 mag from those derived from both the NGC 7790 and PG 0231+051 fields. The discrepancy was confirmed by Nov. 18 data. We found that a similar discrepancy of the $V$ band zero-points for NGC 7654 was also found by Pandey et al. (2001). In detail, however, their magnitudes were not consistent with ours, but $\sim 0.09$ mag fainter. From Dr Stetson (2002, priv. comm.) we learned that the $V$-band calibration for NGC 7654 was based on a single observing run (from the Isaac Newton Group archive). Therefore, we decided not to use this cluster for the calibration. We used the multiple observations of PG $0231+051$ to obtain the atmospheric extinction corrections, finding values consistent with those expected. We looked for colour terms, but found that they were required only for the $B$ band.

For the 60 -inch $4 \mathrm{U} 0142+61$ images, we measured magnitudes using point-spread-function fitting, as implemented in the DAOPHOT II package. We determined aperture corrections using selected, isolated stars, and applied these and the photometric calibration discussed above to determine calibrated magnitudes for stars in the field. 
Next, we cross-identified stars on the 60-inch and Keck images. For the 60-inch 2000 July $V, R$ and $I$-band images, we found 130, 230 and 29 stars that are not overexposed on short Keck images of 1994 October $(V)$ and 1999 September $(R$ and $I$ ). Similarly, 104 and 57 stars in $B$ and $V$, respectively, were found on the 60-inch November 19 images, that are not overexposed on our 2000 December $(B)$ and $1994(V)$ band Keck images. For all Keck images, we obtained instrumental magnitudes using point-spread-function fitting, and we calibrated these directly using the 60 -inch photometry. We found that no colour terms were required for any band. For the $V$ band, we have two independent calibrations. We have used that of 2000 July, since it is based on twice as many comparison stars. For completeness, though, we note that the two $V$-band calibrations differ by less than 0.02 mag. We estimate the uncertainty in the transformation from Palomar to Keck to be about $0.02 \mathrm{mag}$.

Finally, we transferred the photometry from short to long Keck exposures using sets of stars in common. We found that in this last step, in Paper I we had made an error for the $R$ band, causing the $R$-band magnitudes to be 0.08 mag too faint. We estimate that the final uncertainties in the zero points are $0.03 \mathrm{mag}$ in all bands.

\subsection{Final photometry}

In Table 3, we list our calibrated optical and infrared photometry for $4 \mathrm{U} 0142+61$, as well as for all other objects in the field that were detected on at least two of three infrared mosaics.

We list weighted mean magnitudes in all bands, assuming $K=K_{\mathrm{s}}$ and including the results of Paper I. For all stars but $4 \mathrm{U} 0142+61$, we excluded the $B$ band results from September 2000, because it was imaged with a different chip, with possibly different response. The coordinates were derived from the $K$-band image, using astrometry relative to the 1999 Keck $I$-band image, for which we obtained an astrometric solution relative to the USNO-A2.0 catalog in Paper I.

In the $B$-band, we did not detect $4 \mathrm{U} 0142+61$ in either the September or the December data sets. Since the two sets are approximately of equal quality, we have added the average images of both nights. In the average, we find marginal $(2 \sigma)$ evidence for a $B=28.1 \pm 0.5 \mathrm{mag}$ object at the AXP position. The 3- $\sigma$ limit is $B>27.4$; this was determined using simulations similar to those of Hulleman et al. (2000b).

\section{Analysis}

\subsection{Distance and reddening}

There are few constraints on the distance and reddening to $4 \mathrm{U} 0142+61$, which makes the interpretation of the observations rather uncertain. Using stars at similar lines of sight as $4 \mathrm{U} 0142+61$, one can try to determine the run of reddening as a function of distance, and use this to constrain the distance to the source.

At relatively large scales, $4 \mathrm{U} 0142+61$ lies in the general direction of Cas OB8, a somewhat ill-defined OB association, composed of a number of young open clusters (Garmany \& Stencel 1992). The closest clusters are NGC 654 and NGC 663,

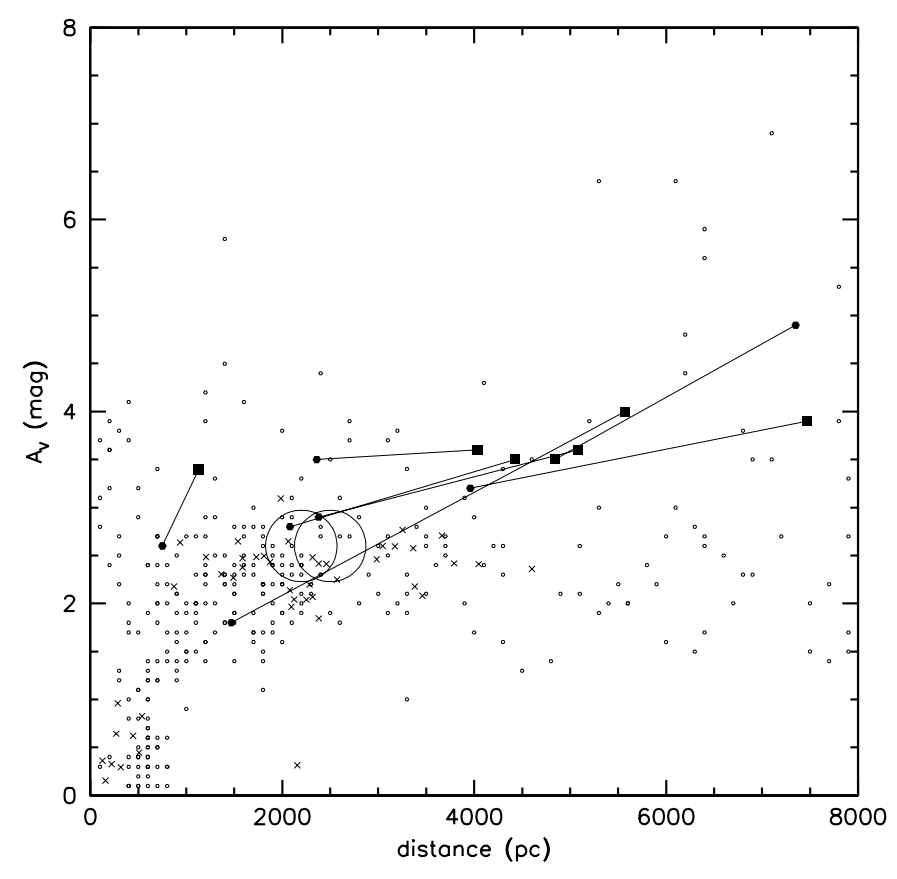

Fig. 3. Reddening as a function of distance, derived by fitting the photometry of stars to stellar models. Big filled squares and hexagons denote the fits to the Keck photometry using the models of Baraffe et al. (1998) and Girardi et al. (2000), respectively, and the small open circles fits to the Palomar photometry, using the models of Girardi et al. (2000). Also shown are the values inferred from the nearby open clusters NGC 654 and NGC 663 (big open circles). Finally, the crosses indicate the galactic extinction as derived by Neckel et al. (1980), using both spectrometric and photometric data of stars for an even larger field (several degrees), encompassing ours.

the centers of which are 17 and $30^{\prime}$ from the line of sight to $4 \mathrm{U} 0142+61$, respectively. Phelps \& Janes (1994) determined $(H W H M)$ radii of 1.39 and 2!69, from a Gaussian fit to the cumulative stellar distributions, and ages of 8-25 and 12-25 Myrs, by fitting stellar isochrones, for NGC 654 and NGC 663, respectively ${ }^{3}$.

The inferred distances for NGC 654 and NGC 663 are $2.5 \mathrm{kpc}$ and $2.2 \mathrm{kpc}$, respectively. The mean reddening for both is $E(B-V)=0.85\left(A_{V}=2.6\right.$; e.g., Stone 1977; Phelps \& Janes 1994), although considerable scatter is observed, $E(B-V)=$ $0.7-1.2\left(A_{V}=2.2-3.7\right)$. Part of the reddening can be attributed to two molecular clouds, NGC 663A and NGC 663C, at $d \sim 3.1$ and $d \sim 1.1 \mathrm{kpc}$ respectively (Leisawitz 1990). Leisawitz et al. (1989) estimate the extinction due to clouds A (C) is of order $\lessgtr 0.9$ (2.2) mag. All these values are lower than the reddening $A_{V}=5.1 \pm 0.1$ to $4 \mathrm{U} 0142+61$ inferred from its X-ray column density of $N_{\mathrm{H}}=9.1 \times 10^{21} \mathrm{~cm}^{-2}$ (Patel et al. 2003) (here, we used the relation $N_{\mathrm{H}}=1.79 \pm 0.03 \times 10^{21} A_{V}$; Predehl \& Schmitt 1995). This suggests that $4 \mathrm{U} 0142+61$ is at a distance $d \gtrsim 2.5 \mathrm{kpc}$, unless it suffers from local absorption. Israel et al. (1994) suggested the latter was the case, and that part of the absorption to $4 \mathrm{U} 0142+61$ was due to the local dark cloud NGC 663H (LDN 1337 Leisawitz et al. 1989; Lynds 1962),

${ }^{3}$ Detailed information about these and other open clusters is available at http://obswww. unige.ch/webda/navigation.html 
which is close to the line of sight and at a distance less than $1 \mathrm{kpc}$.

We have tried to use the stars in our images to make a more local estimate of the amount of interstellar reddening as a function of distance, by fitting the photometry to stellar models. The results are shown in Fig. 3 (see Hulleman 2003, for a detailed discussion). From the Figure, we conclude that the reddening along the line of sight follows generally what is inferred from the open clusters and hot stars, with no evidence for local absorption due to the dark cloud NGC $663 \mathrm{H}$. Within the first kpc, there is a fairly rapid rise to $A_{V}=2$, then the extinction levels off, to $A_{V} \simeq 2.5$ at $2.5 \mathrm{kpc}$, like the open clusters. Beyond that, the Keck data suggest a further slow rise to $A_{V} \simeq 3.5$ at $d=5 \mathrm{kpc}$. The reddening never exceeds $A_{V} \simeq 5$, which is consistent with the limit to the total Galactic reddening of $A_{V} \lesssim 5$, inferred from the observed $R-I$ colour of a background galaxy in Paper I. We must stress however, that the fits to stellar models generally allow multiple solutions, and that, furthermore, we cannot distinguish between giants and main-sequence stars. The latter is responsible for the points at small distance with $2.5 \lesssim A_{V} \lesssim 4$; these are the reddest Palomar stars; they are almost certainly giants at larger distance.

Taken at face value, Fig. 3 confirms the suggestion that $4 \mathrm{U} 0142+61$ is located beyond $5 \mathrm{kpc}$, given the high reddening of $A_{V}=5.1 \pm 0.1$ inferred from its X-ray column density. A puzzle, however, is posed by its dust scattering halo. Using ROSAT observations, White et al. (1996) found that the halo only contributes half as much flux as one would predict based on the column density. Intriguingly, for half the reddening, 4U $0142+61$ would be situated at roughly the same distance as the open clusters and the Cas OB8 association. If so, it might have been born in this association.

A mismatch between the reddening estimates from dustscattering halo and column density and could arise from an error in either. The dust-scattering halo would be smaller for a given amount of reddening if the dust absorption were distributed inhomogeneously along the line of sight. Indeed, from Fig. 3, it follows that much of the reddening occurs in the first kpc.

The reddening inferred from the column density depends crucially on whether the assumed spectral model is correct. The model generally used is the sum of a power-law and a black-body component; if in the true spectrum, the powerlaw does not extend to lower energies (or has a break), then one would overestimate $N_{\mathrm{H}}$. This can be verified using X-ray spectroscopy, by directly measuring the absorption edges. In the Chandra spectrum (Juett et al. 2002), an interstellar Neon edge is present. The depth of this features gives an equivalent $N_{\mathrm{H}}=1.6_{-0.5}^{+0.7} \times 10^{22} \mathrm{~cm}^{-2}$ (Juett 2002, priv. comm.). This indicates a higher rather than a lower extinction, but clearly the large uncertainty precludes a definite answer. Below, we will continue to use the high value of the column density and reddening, although we will mention what would be the effect of reduced values on the optical/infrared spectral energy distribution.

Finally, we would be amiss not to note that a possible similar mismatch between column density and reddening may be present for $1 \mathrm{E} 2259+586$. While from $N_{\mathrm{H}}=9.3 \times 10^{21}$
(Patel et al. 2001), one infers a reddening $A_{V}=5.2$, values between $A_{V}=2.5$ and 3.7 are inferred from measurements of optical filaments of its associated supernova remnant CTB 109 (Fesen \& Hurford 1995).

\subsection{The spectral energy density}

In Fig. 4 we have drawn the spectral energy density of AXP $4 \mathrm{U} 0142+61$ from radio (VLA, $<0.27 \mathrm{mJy}$ at $5 \sigma$ Gaensler et al. 2001) to X-ray (ASCA, White et al. 1996). Both observed and dereddened fluxes are shown, using a reddening of $5.1 \mathrm{mag}$ as derived from the X-ray absorption. Also shown are blow-ups of the dereddened optical and near infrared fluxes, for two choices of reddening, $A_{V}=2.6$ and $A_{V}=5.1$ (see previous section).

While our observations clearly indicate that the infrared flux of the AXP is variable, there is no evidence for variability of the optical fluxes. In particular, all three $R$ band observations yielded the exact same result, $R=24.9$ and we obtain a 2- $\sigma$ limit of $0.09 \mathrm{mag}$ on any variability in $R$. Therefore, we draw the average $V, R$ and $I$-band fluxes over all observations. For $B$, we use the 2- $\sigma$ detection, but draw an downward arrow to remind the reader that it is perhaps better seen as an upper limit (see Sect. 2.3).

From the figure it is clear that the optical/infrared spectral energy distribution cannot be represented by any function that varies only smoothly with frequency, such as a Rayleigh-Jeans tail, but rather that there are spectral features. Particularly striking is the cut-off between the $B$ and $V$ bands. We are fairly certain that this is a real spectral feature, and not due to variability. This is because the optical fluxes did not show any variability and because observations in $R$ and $B$ were taken almost simultaneously in December 2000.

\section{Comparison with other AXPs and predictions}

It is interesting to compare our results for $4 \mathrm{U} 0142+61$ with those for other AXP for which counterparts have been proposed: 1E 2259+586 (Hulleman et al. 2001), 1E 1048.1-5937 (Wang \& Chakrabarty 2002; Israel et al. 2002a) and 1RXS J170849.0-400910 (Israel et al. 2003).

As for $4 \mathrm{U} 0142+61$, the identification of the counterparts to $1 \mathrm{E} 2259+586$ and $1 \mathrm{E} 1048.1-5739$ seems secure. Kaspi et al. (2003) observed 1E 2259+586 just three days after SGRlike X-ray bursts were detected in regular RXTE monitoring observations, and found it to be $1.3 \mathrm{mag}$ brighter in $K_{\mathrm{s}}$ than it was in its discovery images (Hulleman et al. 2001). 1E 1048.1-5739 was discovered as a relatively bright source by Wang \& Chakrabarty (2002), with $K=19.4$, but about a month earlier, it had been undetectable, with $K>20.7$ (Israel et al. 2002a). In this case, no SGR-like bursts were observed in between the infrared measurements of 1E 1048.1-5937, but they may well have been missed given the sparse coverage. The source did show two bursts some months before the infrared measurements were made (Gavriil et al. 2002), which may indicate it was in an active phase. At present, the simplest hypothesis seems to be that also for this source the infrared brightening is related to X-ray burst activity. 


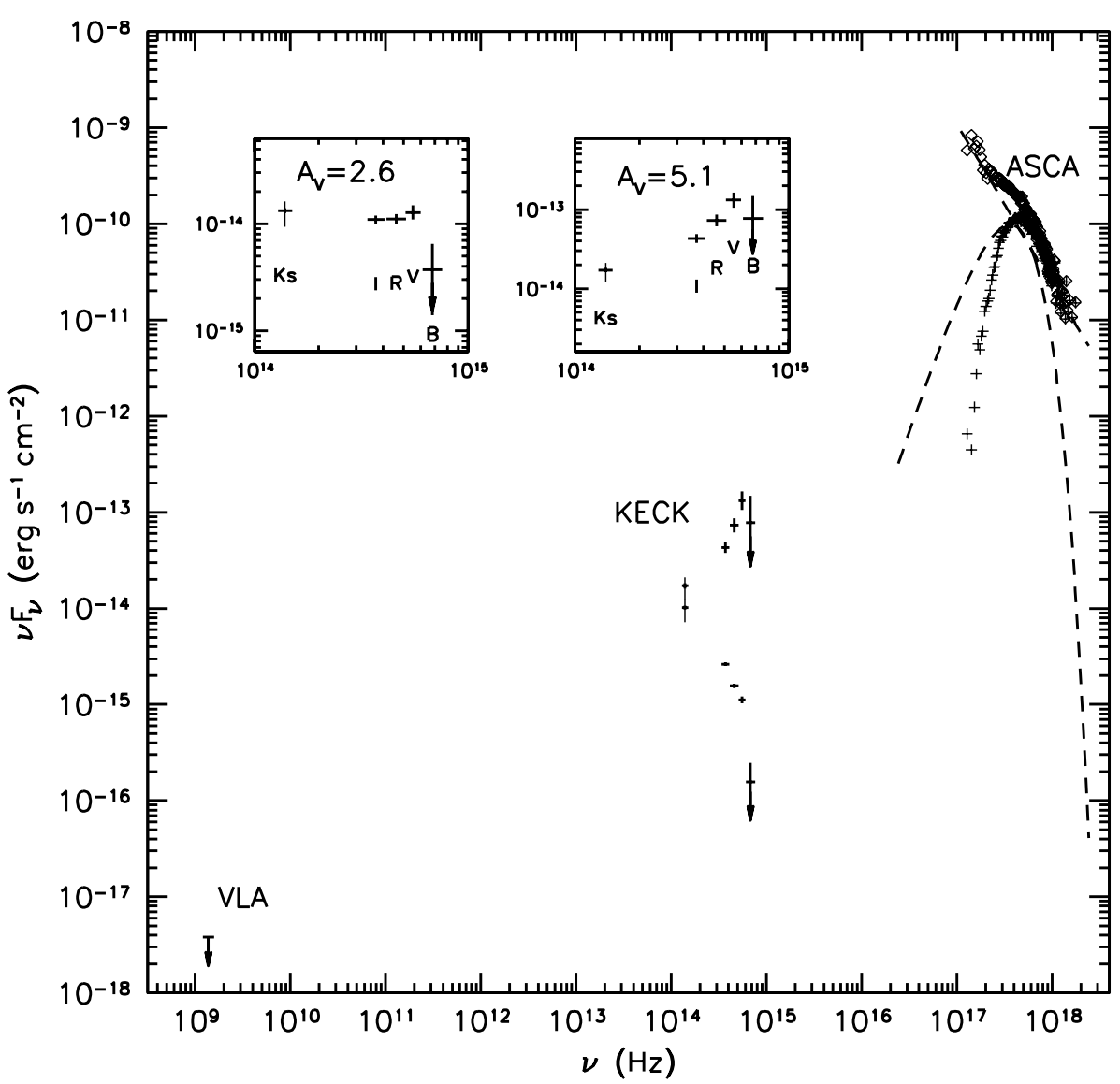

Fig. 4. The spectral energy distribution of AXP 4U 0142+61. Shown are, from left to right, the $1.4 \mathrm{GHz}$ flux limit (VLA, Gaensler et al. 2001), observed and dereddened optical measurements (Keck, this work) and observed and de-extincted X-ray fluxes (ASCA, White et al. 1996). For the dereddening, we used $A_{V}=5.1 \mathrm{mag}$, as inferred from the $\mathrm{X}$-ray absorption. The $B$ band point is the 2- $\sigma$ detection. A downward arrow is drawn as a reminder that this detection is marginal. For the $K_{\mathrm{s}}$ band point we have used the mean magnitude, with thin lines indicating the amount of variability in the form of minimum and maximum fluxes observed. The insets are blow-ups of the dereddened optical/infrared spectrum for two different choices of reddening (see text for details).
For 1RXS J170849.0-400910 the case is less clear. Israel et al. (2003) discovered two relatively faint infrared objects within the Chandra $00^{\prime} 8$ error circle, with $\left(H, K^{\prime}\right)_{\mathrm{A}}=18.85 \pm$ $0.05,17.53 \pm 0.02,\left(H, K^{\prime}\right)_{\mathrm{B}}=20.43 \pm 0.07,20.00 \pm 0.08$ and $J_{\text {sum }}=20.9 \pm 0.1$. Based on its colours, assuming $J_{\mathrm{A}}=J_{\text {sum }}$, they proposed that object $\mathrm{A}$ is the counterpart.

Comparing 4U 0142+61 with 1E 2259+586, the only other AXP with a secure counterpart for which infrared measurements in "quiescence" (i.e. outside burst intervals) are available, we find that the sources share a very similar infrared to $\mathrm{X}$-ray flux ratio. Both the X-ray and the $K_{\mathrm{S}}$ band fluxes for $4 \mathrm{U} 0142+61$ are 4 times larger than those of $1 \mathrm{E} 2259+586$. Interestingly, the $4 \mathrm{U} 0142+61$ and $1 \mathrm{E} 2259+586$ are also very similar in their timing and spectral properties, having similar values of period, period derivative, black-body temperature, power-law index, and thermal fraction of the X-ray flux.

Do all AXPs (and perhaps also SGRs) have similar infrared to X-ray flux ratios? To explore this, we list expected $R$ and $K_{\mathrm{s}}$-band magnitudes in Table 4 , assuming that all magnetars have the same intrinsic X-ray to optical/infrared flux ratio. In Table 4, we also summarise the X-ray spectral properties and lists the observed optical/infrared magnitudes or limits. For all sources, we estimated the reddening $\left(A_{V}\right)$ using the relation $A_{V} / N_{\mathrm{H}}=5.6 \times 10^{-22}$ (Predehl \& Schmitt 1995).

As can be seen from the table, if 1E 1048.1-5937 had a similar infrared to X-ray flux ratio, it would have $K_{\mathrm{s}}=24 \mathrm{mag}$. This is consistent with the upper limit of Israel et al. (2002a), but would imply that it could brighten by 5 mag to match the $K_{\text {s }}=19.3$ observed by Wang \& Chakrabarty (2002). This seems somewhat excessive, and may indicate that, rather, the infrared to X-ray flux ratio is larger, perhaps related to the higher black body temperature and a harder power-law tail (Table 4), and/or the much less stable timing behaviour (Kaspi et al. 2001).

1RXS J170849.0-400910 offers another way to test our predictions. Indeed, if, as Israel et al. (2003) propose, object A is the counterpart to 1RXS J170849.0-400910, our assumption of similar infrared to X-ray flux ratios is wrong. However, if instead object $B$ turns out to be the counterpart, the difference with the predicted magnitude is not so large. We dare not draw conclusions until it is sorted out which of the two candidates is the counterpart.

Clearly, to further our understanding of AXPs (and SGRs), it is crucial to find more counterparts. From the table, it is clear that only the limits for AXP 1048.1-5937 (R), SGR 0526-66 $(R)$ and SGR $1900+14\left(K_{\mathrm{S}}\right)$ are anywhere close to the predicted magnitudes. Furthermore, we see that the prospect of finding new counterparts are best in the near-infrared, which is not surprising given the high extinction to the sources. It should be possible to find at least one or two more counterparts with NICMOS on HST or perhaps one of the larger groundbased telescopes, especially when equipped with adaptive optics. Particularly interesting is the possibility of detecting a SGR: from our estimates, it seems that with a slightly deeper HST image, for instance with ACS, the counterpart to SGR 0526-66 could probably be found. Given the amount of variability seen in 1 E $2259+586$, it would pay to try to observe in times of burst activity. 
Table 4. X-ray spectral results and observed and predicted magnitudes of AXPs.

\begin{tabular}{|c|c|c|c|c|c|c|c|c|c|}
\hline \multirow[b]{2}{*}{ object } & \multirow[b]{2}{*}{$\begin{array}{c}N_{\mathrm{H}} \\
\left(10^{21} \mathrm{~cm}^{-2}\right)\end{array}$} & \multicolumn{2}{|c|}{$\ldots \ldots$ X-ray data $\ldots .}$. & \multirow[b]{2}{*}{$\begin{array}{c}f_{\mathrm{X}}^{\text {unabs }} \\
\left(\mathrm{erg} \mathrm{s}^{-1} \mathrm{~cm}^{-2}\right)\end{array}$} & \multicolumn{2}{|c|}{.... Opt./IR . . . . } & \multicolumn{2}{|c|}{ Predictions . } & \multirow[b]{2}{*}{ ref. } \\
\hline & & $\begin{array}{c}k T \\
(\mathrm{keV})\end{array}$ & $\alpha^{a}$ & & $\begin{array}{c}R \\
\text { (mag) }\end{array}$ & $\underset{(\mathrm{mag})}{K_{\mathrm{s}}}$ & $\underset{(\mathrm{mag})}{R}$ & $\begin{array}{c}K_{\mathrm{s}} \\
(\mathrm{mag})\end{array}$ & \\
\hline $\operatorname{AXP} 0142^{b}$ & $9.1 \pm 0.2$ & $0.470 \pm 0.008$ & $3.40 \pm 0.04$ & $4.8 \times 10^{-10}$ & 24.9 & 20.1 & $\ldots$ & $\ldots$ & 1,2 \\
\hline $\operatorname{AXP} 2259^{b}$ & $9.3 \pm 0.3$ & $0.412 \pm 0.006$ & $3.6 \pm 0.1$ & $1.2 \times 10^{-10^{c}}$ & $>26.4$ & $20.4-21.7$ & 27 & 22 & $3,4,5$ \\
\hline $\operatorname{AXP} 1048^{b}$ & $10.4 \pm 0.8$ & $0.63 \pm 0.03$ & $2.9 \pm 0.2$ & $1.4 \times 10^{-11^{c}}$ & $>24.8$ & $19.3,>20.7$ & 29 & 24 & $6,7,8$ \\
\hline AXP $1708^{b}$ & $14.2 \pm 1.5$ & $0.46 \pm 0.03$ & $2.62 \pm 0.17$ & $1.2 \times 10^{-10}$ & $>20$ & $18.3 ?$ & 29 & 22 & $9,10,11$ \\
\hline AXP Kes73 & $\sim 20$ & $\ldots$ & $3.0 \pm 0.2$ & $6.3 \times 10^{-11}$ & $\gtrsim 23.5$ & $\gtrsim 17$ & 30 & 23 & 12,13 \\
\hline $\mathrm{SMC} \mathrm{AXP}^{b}$ & $1.4 \pm 0.2$ & $0.41 \pm 0.01$ & $\ldots$ & $6.9 \times 10^{-13^{c}}$ & $\ldots$ & $\ldots$ & 28 & 27 & 14 \\
\hline SGR $0526^{b}$ & $5.5 \pm 0.2$ & $0.53 \pm 0.06$ & $3.14 \pm 0.08$ & $3.42 \times 10^{-12}$ & $\gtrsim 26^{d}$ & $\ldots$ & 28 & 25 & 15,16 \\
\hline SGR $1900^{b}$ & $24 \pm 2$ & $0.46 \pm 0.05$ & $2.4 \pm 0.2$ & $2.5 \times 10^{-11}$ & $\ldots$ & $>20.8$ & 35 & 24 & 17,18 \\
\hline SGR $1806^{b}$ & $63 \pm 5$ & $\ldots$ & $1.95 \pm 0.10$ & $3.0 \times 10^{-11}$ & $\ldots$ & $16.7 ?$ & 53 & 26 & 19,20 \\
\hline SGR $1627^{b}$ & $77 \pm 8$ & $\ldots$ & $2.5 \pm 0.2$ & $1.5 \times 10^{-11}$ & $\ldots$ & $\ldots$ & 60 & 28 & 21 \\
\hline
\end{tabular}

a Power-law photon index, ${ }^{b}$ full names are 4U 0142+61, 1E 2259+586, 1E 1048.1-5937, 1RXS J170849.0-400910, CXOU J0110043.1-721134, SGR 0526-66, SGR 1900+14, SGR 1806-20 and SGR 1627-41, ${ }^{c}$ we have used PIMMS to convert the X-ray fluxes to the $0.5-10 \mathrm{keV}$ range, ${ }^{d}$ interpolation between observed magnitudes in HST bands F547M and F814W.

References: 1: Patel et al. (2003), 2: this work, 3: Patel et al. (2001), 4: Hulleman et al. (2001), 5: Kaspi et al. (2003), 6: Tiengo et al. (2002), 7: Wang \& Chakrabarty (2002), 8: Israel et al. (2002a), 9: Israel et al. (2001), 10: Israel et al. (1999), 11 Israel et al. (2003), 12: Vasisht \& Gotthelf (1997), 13: Mereghetti et al. (2001), 14: Lamb et al. (2002), 15: Kulkarni et al. (2003), 16: Kaplan et al. (2001), 17: Kouveliotou et al. (2001), 18: Kaplan et al. (2002), 19: Mereghetti et al. (2000), 20: Eikenberry et al. (2001), 21: Woods et al. (1999).

\section{Discussion}

While the results presented in Paper I favored the magnetar model, they still allowed for a truncated accretion disk and a massive white dwarf. The (truncated) accretion disk gives a simple explanation for the optical to X-ray flux ratio. In this model, the optical flux is due to reprocessing of the X-ray flux by the disk. However, as already argued by Kern \& Martin (2002) it cannot explain the optical pulsations. Moreover, the optical spectrum is not expected to show the spectral features we observe, since it should consist of thermal radiation coming from different parts of the disk.

For a massive magneto-white dwarf the situation is less clear. Although the spectrum is no longer well represented by a Rayleigh Jeans tail, there is the possibility that the feature at $B$ is due to absorption at the electron cyclotron frequency for magnetic field of a few times $10^{8}$ Gauss. However, it is unclear what could cause the observed infrared excess and variability at $K$ in such a white dwarf. Furthermore, the observations of glitches in the timing Kaspi et al. (2003) as well as the X-ray bursts (Gavriil et al. 2002; Kaspi et al. 2003) seem hard to square with AXPs being white dwarfs.

This leaves us with the magnetar model. In a magnetar magnetosphere the magnetic field is thought to be twisted, due to the presence of large-scale currents in the magnetosphere. These currents are induced by motions of the footpoints of the magnetic field as a result of the unwinding of the internal magnetic field (Thompson et al. 2002). Assuming a mildly twisted magnetic field with $B_{\mathrm{s}}=1.3 \times 10^{14} \mathrm{G}$ at the surface of the neutron star, we find that at the transition radius $R_{\text {cool }}$, where the spontaneous cyclotron cooling time of the ions equals their transit time along the magnetic field (Thompson et al. 2002), the proton cyclotron frequency, $v_{\text {cyc }}^{\mathrm{i}}=\frac{Z e B}{2 \pi A m_{\mathrm{p}} c}=$ $6 \times 10^{17}\left(\frac{B}{10^{14} \mathrm{G}}\right) \mathrm{Hz}^{4}$, and its harmonic correspond to the $H$ and $V$ bands. Could the spectral feature between $B$ and $V$ be due to a peak at $V$ ? If so, one would also expect a peak at $H$. Observations could easily confirm such a second peak.

The observed (infrared) variability of AXPs 1E 2259+586 and 1E 1048.1-5937 (Kaspi et al. 2003; Israel et al. 2002a) is probably related to the occurence of SGR-like X-ray bursts (see Sect. 4). We have now also detected infrared variability of AXP 4U 0142+61. Therefore it seems likely that the source emits similar bursts. These may easily have gone undetected in the past given the sparse temporal coverage of X-ray observations of the source. Again, this can be checked observationally, although it would probably require simultaneous monitoring using both X-ray and infrared telescopes.

Acknowledgements. We thank David Kaplan and Brian Jacoby for obtaining the 2000 July and November Palomar 60 inch data, and David Kaplan and George Djorgovski for obtaining the September and December Keck LRIS data. Part of this work was done while the authors were visiting the Institute for Theoretical Physics at UCSB, which is supported by NSF grant PHY 99-07949. F.H. thanks the Netherlands Organization for Scientific Research (NWO) and the

\footnotetext{
${ }^{4}$ Note that the ion cyclotron feature is at X-ray energies for $r \sim$ $R_{\mathrm{NS}}$. Chandra HETG observation, failed to reveal a cyclotron feature Juett et al. (2002) for $1.9 \times 10^{14} \lesssim B \lesssim 9.8 \times 10^{14} \mathrm{G}$. While this seemed inconsistent with earlier predictions (e.g. Zane et al. 2001), recent work has shown that then feature may actually be difficult to observe, as the line is suppressed by effects due to vacuum polarisation (Ho \& Lai 2003).
} 
Leids Kerkhoven Bosscha Fund for their grants, which made this visit possible. M.H.v.K. acknowledges support from a fellowship of the Royal Netherlands Academy of Arts and Sciences.

\section{References}

Baraffe, I., Chabrier, G., Allard, F., \& Hauschildt, P. H. 1998, A\&A, 337,403

Coe, M. J., \& Pightling, S. L. 1998, MNRAS, 299, 223

Duncan, R. C., \& Thompson, C. 1992, ApJ, 392, L9

Eikenberry, S. S., Garske, M. A., Hu, D., et al. 2001, ApJ, 563, L133

Fesen, R. A., \& Hurford, A. P. 1995, AJ, 110, 747

Gaensler, B. M., Slane, P. O., Gotthelf, E. V., \& Vasisht, G. 2001, ApJ, 559, 963

Garmany, C. D., \& Stencel, R. E. 1992, A\&AS, 94, 211

Gavriil, F. P., \& Kaspi, V. M. 2002, ApJ, 567, 1067

Gavriil, F. P., Kaspi, V. M., \& Woods, P. M. 2002, Nature, 419, 142

Girardi, L., Bressan, A., Bertelli, G., \& Chiosi, C. 2000, A\&AS, 141, 371

Heyl, J. S., \& Hernquist, L. 1998, MNRAS, 300, 599

Ho, W. C. G., \& Lai, D. 2003, MNRAS, 338, 233

Hulleman, F. 2003, Anomalous X-ray Pulsars at optical and infrared wavelengths, Ph.D. Thesis, Utrecht University

Hulleman, F., Tennant, A. F., van Kerkwijk, M. H., et al. 2001, ApJ, 563, L49

Hulleman, F., van Kerkwijk, M. H., \& Kulkarni, S. R. 2000a, Nature, 408, 689

Hulleman, F., van Kerkwijk, M. H., Verbunt, F. W. M., \& Kulkarni, S. R. 2000b, A\&A, 358, 605

Israel, G., Oosterbroek, T., Stella, L., et al. 2001, ApJ, 560, L65

Israel, G. L., Covino, S., Perna, R., et al. 2003, ApJ, 589, L93

Israel, G. L., Covino, S., Stella, L., et al. 1999, ApJ, 518, L107

Israel, G. L., Covino, S., Stella, L., et al. 2002a, ApJ, 580, L143

Israel, G. L., Mereghetti, S., \& Stella, L. 1994, ApJ, 433, L25

Israel, G. L., Mereghetti, S., \& Stella, L. 2002b, MmSAI, 73, 465

Juett, A. M., Marshall, H. L., Chakrabarty, D., \& Schulz, N. S. 2002, ApJ, 568, L31

Kaplan, D. L., Kulkarni, S. R., Frail, D. A., \& van Kerkwijk, M. H. 2002, ApJ, 566, 378

Kaplan, D. L., Kulkarni, S. R., van Kerkwijk, M. H., et al. 2001, ApJ, 556, 399

Kaspi, V. M., Gavriil, F. P., Chakrabarty, D., Lackey, J. R., \& Muno, M. P. 2001, ApJ, 558, 253

Kaspi, V. M., Gavriil, F. P., Woods, P. M., et al. 2003, ApJ, 588, L93

Kern, B., \& Martin, C. 2002, Nature, 417, 527

Kouveliotou, C., Tennant, A., Woods, P. M., et al. 2001, ApJ, 558, L47

Krisciunas, K., Sinton, W., Tholen, K., et al. 1987, PASP, 99, 887
Kulkarni, S. R., Kaplan, D. L., Marshall, H. L., et al. 2003, ApJ, 585, 948

Lamb, R. C., Fox, D. W., J., M. D., \& Prince, T. A. 2002, ApJ, 574, L29

Leisawitz, D. 1990, ApJ, 359, 319

Leisawitz, D., Bash, F. N., \& Thaddeus, P. 1989, ApJS, 70, 731

Lynds, B. T. 1962, ApJS, 7, 1

Matthews, K., \& Soifer, B. T. 1994, in Astronomy with Arrays, The Next Generation, ASSL 190, 239

Mereghetti, S., Cremonesi, D., Feroci, M., \& Tavani, M. 2000, A\&A, 361,240

Mereghetti, S., Israel, G. L., \& Stella, L. 1998, MNRAS, 296, 689

Mereghetti, S., Mignani, R. P., Covino, S., et al. 2001, MNRAS, 321, 143

Neckel, T., Klare, G., \& Sarcander, M. 1980, A\&AS, 42, 251

Oke, J. B., Cohen, J. G., Carr, M., et al. 1995, PASP, 107, 375

Özel, F. 2001, ApJ, 563, 276

Pandey, A. K., Nilakshi, Ogura, K., Sagar, R., \& Tarusawa, K. 2001, A\&A, 374, 504

Patel, S. K., Kouveliotou, C., Woods, P. M., et al. 2001, ApJ, 563, L45

Patel, S. K., Kouveliotou, C., Woods, P. M., et al. 2003, ApJ, 587, 367

Pavlov, G. G., Sanwal, D., Garmire, G. P., \& Zavlin, V. E. 2002, in

Neutron Stars in Supernova Remnants, ASP Conf. Ser., ed. P. O.

Slane, \& B. M. Gaensler, 247 [astro-ph/0112322]

Persson, S. E., Murphy, D. C., Krzeminski, W., Roth, M., \& Rieke, M. J. 1998, AJ, 116, 2475

Phelps, R. L., \& Janes, K. A. 1994, ApJ, 90, 31

Predehl, P., \& Schmitt, J. H. M. M. 1995, A\&A, 293, 889

Reid, C. A., Johnston, M. D., Bradt, H. V., et al. 1980, AJ, 85, 1062

Stetson, P. B. 1987, PASP, 99, 191

Stetson, P. B. 2000, PASP, 112, 925

Stone, R. C. 1977, A\&A, 54, 803

Thompson, C., \& Duncan, R. C. 1996, ApJ, 473, 322

Thompson, C., Lyutikov, M., \& Kulkarni, S. 2002, ApJ, 574, 332

Tiengo, A., Göhler, E., Staubert, R., \& Mereghetti, S. 2002, A\&A, 383,182

Vasisht, G., \& Gotthelf, E. V. 1997, ApJ, 486, L129

Wang, Z., \& Chakrabarty, D. 2002, ApJ, 579, L33

White, N. E., Angelini, L., Ebisawa, K., Tanaka, Y., \& Ghosh, P. 1996, ApJ, 463, L83

White, N. E., Mason, K. O., Giommi, P., et al. 1987, MNRAS, 226, 645

Wilson, C. A., Dieters, S., Finger, M. H., Scott, D. M., \& van Paradijs, J. 1999, ApJ, 513, 464

Woods, P. M., Kouveliotou, C., van Paradijs, J., et al. 1999, ApJ, 519, L139

Zane, S., Turolla, R., Stella, L., \& Treves, A. 2001, ApJ, 560, 384 